\title{
Where to Build It?
}

\section{Don Dennison}

Published online: 20 December 2013

(C) Society for Imaging Informatics in Medicine 2013

You have an idea for a new application for healthcare. Your options for what kind of environment to build it in may be limited, based on where you are today, but let's explore the pros and cons of different ones. We need to think about the cost to build it, what kind of revenue opportunity there might be, how you will collect proper design input and validate your product, as well as how to transition from development to support.

An Established Vendor refers to a company that has produced one or more applications, has an installed base with a revenue stream, and features $R \& D$, sales, and service and support staff. The organization will have other applications competing for resources and focus.

A Start-up is a company founded for the purpose of building your new application. In addition to building the application, the organization itself must first be developed.

Hospital Developed is about a team of employees (or contractors), and often interns, developing an application for use within their institution, and perhaps others. Developing applications is not the primary purpose of the organization.

\section{Cost to Build}

This relates to the cost to define, develop, test, and launch the application.

\section{Established Vendor}

Costs are typically high as the overhead of the larger organization needs to be accounted for. Also, employees have often been with the company longer, resulting in seniority and higher compensation. Process and tools are often mature but

D. Dennison $(\bowtie)$

Don K Dennison Solutions Inc., Waterloo, ON, Canada

e-mail: don@dondennison.com can be time consuming. Policies may be restrictive and not conducive to innovation and agility. Validating the application can be expensive as travel is usually involved. Often, a combination of internal departments with experienced staff and service providers under contract (e.g., PR firms), along with established policies and processes, is available to launch the product — some savings can be found if launching the product along with others (joint marketing). The product may be marketed to existing contacts within the installed base using low-cost methods, like email distributions and user group meetings.

\section{Start-up}

Costs can be more easily controlled as the organization is developed along with the product. The skill sets of the staff can be optimized to match those needed by the application. Process and tools can be limited to "just enough to get the job done." As employees tend to be new hires, they are often less experienced-and therefore less expensive-than in Established Vendors. Validating the application can be relatively expensive for the Start-up, as travel is often involved. If the market is unfamiliar with the Start-up, some amount of investment is likely necessary in order to develop a corporate brand. Start-ups have the advantage of focusing their launch to a specific profile of customer and can optimize the marketing around how that customer prefers to buy.

Hospital Developed

Hospital staff often have other duties to "subsidize" the costs of development. In some cases, research grants can apply to offset costs. Process ranges from minimal to nonexistent. Tools are often open source, keeping costs to a minimum. Productivity can sometimes be suboptimal if the staff lack experience in professional application development, resulting 
in rework. Validation costs are minimal as users, data, and operating environments are readily available within the institution. Launching the application to the internal user community is very low cost to free.

\section{Revenue Opportunity}

This is about bringing in money_-plain and simple. It can be from application license fees, technical and professional services, support contracts, subscriptions, or other models.

\section{Established Vendor}

Vendors exist for the purpose of generating revenue, so this is obviously a focus. With other complementary applications already available and installed in the market, generating revenue from a new application is easier. Existing customers exist to testify to the company's capability and track record. Market research staff can maximize product positioning and provide input to pricing models and amounts. Services staff will often have a process for defining new service catalog offerings, allowing revenue generation from technical and professional services, in addition to application license revenues. Service and support contract templates, with staff behind them, are defined and available.

\section{Start-up}

Without an established customer base and with a low-cost structure, the Start-up has greater price flexibility than an Established Vendor, though it can often ill afford to "buy" a market by selling at a loss like an Established Vendor with sufficient cash in hand can. Start-ups can also provide innovative licensing models where Established Vendors often struggle to offer and support new models effectively. This can allow Start-ups to be disruptive in a market.

\section{Hospital Developed}

Often, revenue generation is not a focus for a hospital-based group. When it is a priority, the opportunities are often limited. The shift from internal service provider supporting other groups within the same organization to being a service provider in an open and competitive market is not easy and is often where teams fail. Often, after the initial user needs are sated and the research findings published, interest/momentum is lost, and resources are redistributed. Engaging vendor personnel (from an Established Vendor or Start-up organization) with experience in launching a product is recommended. Outsourcing the service and support aspects of the launch may be an option, if an effective service provider partner can be found; however, this approach will likely limit revenue opportunities to only software licensing. If the intellectual property (IP) is innovative enough and properly protected, licensing the technology to other vendors may be an opportunity.

\section{Design Input and Validation}

When developing an application, quality design input and realistic validation are critical aspects. Access to the target user community, data, and operating environment is necessary.

\section{Established Vendor}

With an installed base and broad organization supporting it, design inputs (unmet needs, feature requests, etc.) are often plentiful and varied. Managing these inputs, identifying the most valuable ones, and distilling them to use cases (or user stories, when using Agile development methods) and requirements are a key function of product management. A clear vision with concise design principles proves to be useful in separating signal from noise. Validation partners can typically be found among the installed base of complementary products. Restricting validation partners to a select few customers that represent the target market profile of the product is recommended. If other products or services provided to the installed base are resulting in low customer satisfaction, it may limit the number of customers willing to enter a partnership to validate the new product. It may even result in the new product being offered at a deep discount to compensate for past failed commitments.

\section{Start-up}

Start-ups often partner with a luminary customer early on. These customers provide not only early design input and validation but also, hopefully, revenue (though often the first customer pays little for the solution). They also provide the Start-up with legitimacy among the customer's peers, leading to new opportunities. The first customer must represent the target market profile of the customer for which the product is intended; otherwise, they may steer the product towards addressing non-core market customer needs.

\section{Hospital Developed}

By being based within a hospital environment, the team has access to a deep pool of design inputs, including target users. They also have an innate knowledge of the hospital's own operating environment and what systems designs, such as operating platforms and network configurations, would be accepted. They also have access to all necessary data for design analysis and validation. Assuming that the hospital where the team is based is typical of the target market profile for the product, this 
Table 1 Comparisons for different building environments

\begin{tabular}{lllll}
\hline & Cost to build & $\begin{array}{l}\text { Revenue } \\
\text { opportunity }\end{array}$ & $\begin{array}{l}\text { Design input } \\
\text { and Validation }\end{array}$ & $\begin{array}{l}\text { Operational } \\
\text { support }\end{array}$ \\
\hline Established vendor & ++ & +++ & ++ & +++ \\
Start-up & ++ & ++ & ++ & ++ \\
Hospital developed & + & + & ++ & ++ \\
\hline
\end{tabular}

environment provides an opportunity for deep understanding of important nuances when designing the product and a rapid and ongoing feedback loop in product validation.

\section{Operational Support}

Supporting an application that is in use requires an organization with the appropriate capabilities and capacity, along with an effective process and tools.

\section{Established Vendor}

Most established vendors have a mature organization with defined processes, such as Information Technology Infrastructure Library (ITIL) and tools [1]. Often, Established Vendors have methods and templates to manage service level agreements (SLAs) with customers. Support staff is typically remote, organized in central call centers or distributed in home offices, but some on-site field service staff may be available. Proactive system monitoring may be integrated with the support system, but not always. Depending on the product development plan used, a product may be made up of components developed in different sites in different parts of the world, significantly complicating troubleshooting when problems are escalated to R\&D.
Start-up

In the early days of the organization, delivery and support is provided by R\&D staff, resulting in expert support and rapid response to issues; however, as the installed base grows, staff will find support activities competing with other priorities, such as fulfilling the product roadmap. Often, support processes and tools are immature, but these can be defined to be optimized for the product being developed (vs. a generalized approach often found in an Established Vendor providing several products). Support staff (when hired) is often remote simply due to the high cost of field personnel, but co-locating them with R\&D staff (all in one facility) often results in a deeper knowledge in the support staff and more rapid response time to problem resolution.

\section{Hospital Developed}

Formal support processes and tools are often overlooked when the customer is internal. Formal service level agreements (SLAs) with users and buyers are often not put in place, leading to potential expectation misalignment when problems arise. However, knowledge of the user community is deep, allowing for effective prioritization of issues (spending energy on the most critical problems). Support staff is typically on-site. Internal IT support desk tools ("trouble ticket" tracker, on call schedule, etc.) may be leveraged to avoid the cost and complexity of putting a formal support management system in place.

Fig. 1 Lifecycle for bringing an idea to market

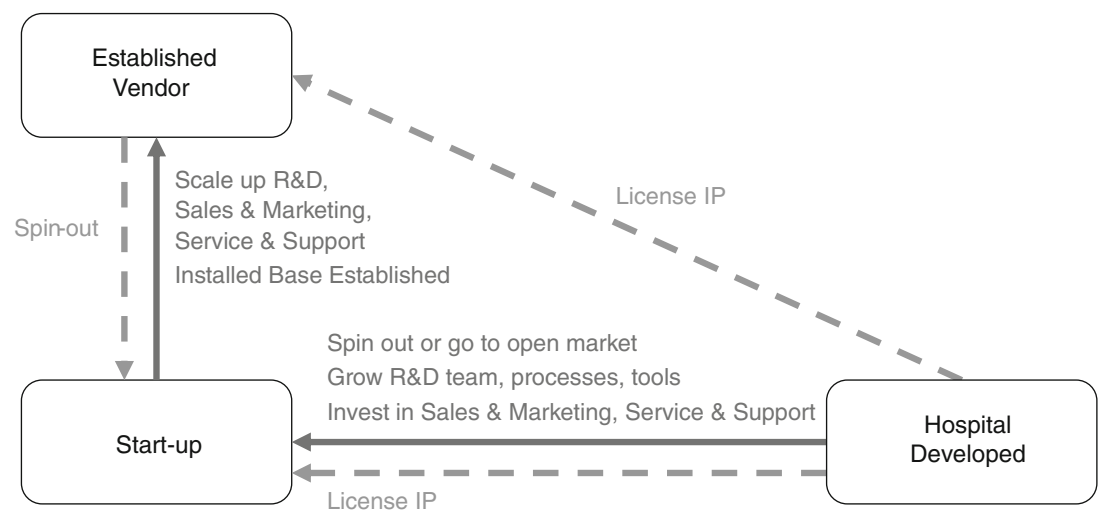




\section{Summary}

Table 1 is a summary comparing all the aspects rated for each environment.

Comparing the Aspects for Each Environment

In general, the Hospital Developed environment has the advantage of direct access to the healthcare stakeholders, whereas the Established Vendor and Start-up are better positioned to commercialize products, though their cost structures are typically higher.

\section{Paths to Growth}

Whichever environment you are in (or choose), as you work to build your team, product, and customer base, you will be faced with choices that will naturally change the starting environment to a new one (Fig. 1).

\section{Analyzing the Paths to Growth}

Though it is possible that an Established Vendor or Start-up could transfer IP to a hospital, it is rare enough that it is not shown.

While the opportunity to license IP from the Hospital Developed environment to the Start-up or Established vendor, these are rare. Commercial entities have to concern themselves with the cost of goods, including licensed IP, and of their solution in order to ensure profits. Often, the IP creators in the Hospital Developed environment have a perception that their innovation is worth much more than the commercial entity is willing to pay.

\section{Additional Resources}

Accelerator centers can be very useful to help Start-ups develop their products and business. They can also be useful for hospital-based teams to become Start-ups. Some healthcare IT-specific incubator and accelerator centers [2] have started to emerge [3].

One trend that has begun to emerge is a practice called DevOps [4], an approach that blends software developer staff and support staff in providing proactive support services. Interestingly, this method can be adopted in any of the environments listed.
Best practices for IT service management and support delivery have been defined as part of ITIL. While the concepts are valuable to understand in any environment, due to the structure, training, and process investment, ITIL is more feasible to implement in an Established Vendor, though some hospital IT staff are starting to adopt ITIL best practices.

\section{Discussion}

This article did not explore the typical or possible methods of establishing or growing a Start-up, as this topic is well documented in general business literature. Venture capital investment in Start-ups focused on healthcare IT is reported to be significant at the time of publication, however.

Time to market was an aspect considered for inclusion in this analysis; however, due to the notable relationship to the type of product built (e.g., a simple mobile app vs. a complex enterprise platform vs. a rich clinical application dependent on as-yet-not-invented algorithms), it was not included. In general, Start-ups have the shortest time to market but face challenges when transitioning to become an Established Vendor. Time to market in Hospital Developed environments has a couple of milestones when considering measuring time to market: when the product is available for use by internal hospital users (ready for operations) and when the product is available for purchase in the open market (commercialized). The latter will mean that many of the challenges of a Start-up identified in this article will apply to the hospital-based team.

Acknowledgments The author would like to thank the valuable contributions and much appreciated encouragement from Paul G. Nagy, PhD, Baltimore, MD.

\section{References}

1. ITIL. Available at http://www.itil-officialsite.com/. Accessed 24 October 2013

2. Start-up Owl: Venture Incubators and Accelerators. Available at http:// startupowl.com/resources/startup-boosters/incubators-andaccelerators/. Accessed 24 October 2013

3. Healthbox announces next startup class. Available at http:/www. healthcareitnews.com/news/healthbox-announces-next-startup-class? single-page=true. Accessed 24 October 2013

4. Top 10 Practices for Effective DevOps. Available at http:/www. drdobbs.com/architecture-and-design/top-10-practices-for-effectivedevops/240149363. Accessed 24 October 2013 